\title{
Thermal Dissociation and Conformational Lock of Superoxide Dismutase
}

\author{
J. Hong, A. A. Moosavi-Movahedi*, H. Ghourchian, M. Amani, M. Amanlou ${ }^{\dagger}$ and F.C. Chilaka \\ Institute of Biochemistry and Biophysics, University of Tehran, Tehran, Iran \\ 'Department of Medicinal Chemistry, Tehran University of Medical Sciences, Tehran, Iran \\ Department of Biochemistry, University of Nigeria, Nsukka, Nigeria
}

Received 9 March 2005, Accepted 5 May 2005

\begin{abstract}
The kinetics of thermal dissociation of superoxide dismutase (SOD) was studied in $0.05 \mathrm{M}$ Tris-HCl buffer at $\mathrm{pH} 7.4$ containing $10^{-4} \mathrm{M}$ EDTA. The number of conformational locks and contact areas and amino acid residues of dimers of SOD were obtained by kinetic analysis and biochemical calculation. The cleavage bonds between dimers of SOD during thermal dissociation and type of interactions between specific amino acid residues were also simulated. Two identical contact areas between two subunits were identified. Cleavage of these contact areas resulted in dissociation of the subunits, with destruction of the active centers, and thus, lost of activity. It is suggested that the contact areas interact with active centers by conformational changes involving secondary structural elements.
\end{abstract}

Keywords: Biochemical calculation, Conformational lock, Contact area, Kinetics, Superoxide dismutase (SOD), Thermal dissociation

\section{Introduction}

Superoxide dismutases ( $\mathrm{Cu}, \mathrm{Zn}$-SODs) have been found in the cytoplasm of all the eukaryotic cells and in the periplasm of several bacterial species (Bannister et al., 1987; Kroll et al., 1995). Eukaryotic $\mathrm{Cu}, \mathrm{Zn}-\mathrm{SODs}$ are homodimers that contain one atom of zinc and one atom of copper per subunit and catalyze the dismutation of the superoxide anion at a

\footnotetext{
Abbreviations: SOD, superoxide dismutase; $\mathrm{T}_{\mathrm{op}}$, optimum temperature; EDTA, ethylenediaminetetracetic acid; Tris, tris(hydroxymethyl) aminomethane; SDS, sodium dodecyl sulfate
}

*To whom correspondence should be addressed.

Tel: +98-21-6403957; Fax: +98-21-6404680

E-mail: moosavi@ut.ac.ir diffusion-limited rate enhanced by electrostatic guidance of the substrate to the active site (Desideri et al., 1992). Cu, ZnSODs possess a very compact structure that is highly resistant to denaturation by urea, SDS and proteolytic degradation. Several factors are thought to contribute to the enzyme stability, including the prosthetic metal ions (Forman and Fridovich, 1973), the intrasubunit disulfide bond (Abernethy et al., 1974), and the close packing of the hydrophobic interface between the subunits and the two halves of the $\beta$ barrel core (Getzoff et al., 1989). Amino acid sequence comparisons and the analysis of the three dimensional structure of the dimeric enzyme from P.leiognathi have shown that the prokaryotic and eukaryotic $\mathrm{Cu}, \mathrm{Zn}-\mathrm{SODs}$ share a conserved ligand stereochemistry and a very similar monomer fold, based on a flattened Greek-key eight-stranded $\beta$-barrel (Bordo et al., 1994; Bourne et al., 1996; Imlay and Imlay, 1996; Battistoni et al., 1996).

Since the discovery of $\mathrm{Cu}, \mathrm{Zn}-\mathrm{SOD}$, there has been serious in understanding how the dimeric structure contributes to the high catalytic efficiency and the remarkable stability of this class of enzymes. All attempts to obtain monomeric $\mathrm{Cu}, \mathrm{Zn}$ SODs by treatments with detergents (Rigo et al., 1978) or site-directed substitutions of hydrophobic residues at the dimer interface (Bertini et al., 1994) provided also enzymes that display very low catalytic activity and gross alterations of spectroscopic properties. These dramatic changes probably reflect changes in the tertiary structure consequent to rearrangements of the solvent-exposed hydrophobic dimer interface.

The properties of interfaces in oligomeric enzymes and their influence on catalytic activity can be studied and explained by two independent methods, involving the use of the structural and kinetics data. Poltorak et al. (1999a; 1999b) reported that, in alkaline phosphatase from different sources, the same results that in both methods are in reasonable agreement. In our previous paper (Moosavi-Nejad et al., 2003), we had put forward a mechanism of Lentil seedling amine oxidase denaturation based on structural data. In 
present study, thermal dissociation and conformational lock in addition to biochemical calculation were used to obtain a putative mechanism for thermal dissociation of superoxide dismutase.

\section{Materials and Methods}

Materials Superoxide dismutase (SOD) from bovine erythrocyte [E.C: 1.15.1.1] was a product of Sigma (S2515). Pyrogallol, Tris, EDTA and $\mathrm{HCl}$ were the products of Merck.

Enzyme assay The activity of superoxide dismutase from bovine erythrocyte was determined by using an improved pyrogallol autoxidation inhibition assay (Marklund and Marklund, 1974; Zou et al., 1986; Luo et al., 1987; Yuan and Gao, 1997). Superoxide $\left(\mathrm{O}_{2} \cdot\right)$ is generated indirectly at alkaline $\mathrm{pH}$ by the action of oxygen on pyrogallol. SOD reacts with the superoxide and this slows down the rate of formation of the o-hydroxy-o-benzoquinone and other polymer products (Liang et al., 2000). One unit of SOD is defined as the amount of enzyme that reduces the rate of autoxidation of pyrogallol by 50\%. (Marklund and Marklund, 1974; Zou et al., 1986; Luo et al., 1987; Yuan and Gao, 1997; Liang et al., 2000).

The assay is performed in $1 \mathrm{ml}$ of $0.05 \mathrm{M}$ Tris- $\mathrm{HCl}$ buffer at $\mathrm{pH}$ 8.2 containing $10^{-4} \mathrm{M}$ EDTA at $27^{\circ} \mathrm{C}$ in a thermostated Shimadzu UV-Vis-3100 spectrophotometer. The standard reaction mixture usually contained a certain concentration pyrogallol, which produces a rate of autoxidation of 0.07 absorbance unit per min measured at $325 \mathrm{~nm}$. Under this condition, the amount of superoxide dismutase required to reduce the rate to 0.035 absorbance unit per min is defined as one unit of activity (Marklund and Marklund, 1974; Zou et al., 1986; Luo et al., 1987; Yuan and Gao, 1997; Liang et al., 2000).

Determination of optimum temperature Optimum temperature $\left(T_{\text {opt }}\right)$ is defined as the maximum temperature at which the activity of the enzyme does not change during the incubation time (Segel, 1995). SOD samples at a concentration of $4.9 \times 10^{-7} \mathrm{M}$ were incubated in multiTem III thermostat (Pharmacia Biotech, Uppsala, Sweden) at different temperatures (from $27-77^{\circ} \mathrm{C}$ ) in $0.05 \mathrm{M}$ Tris$\mathrm{HCl}$ buffers at $\mathrm{pH} 7.4$ containing $10^{-4} \mathrm{M}$ EDTA for 150 minutes, cooled rapidly to $27^{\circ} \mathrm{C}$, and assayed for residual activity.

Thermal dissociation of SOD SOD samples at a concentration of $4.9 \times 10^{-7} \mathrm{M}$, in $50 \mathrm{mM}$ Tris- $\mathrm{HCl}$ buffer at $\mathrm{pH} 7.4$ containing 0.1 mM EDTA, were incubated separately at 75 and $77^{\circ} \mathrm{C}$. At suitable intervals, usually 5 minutes, samples were removed and cooled rapidly to $27^{\circ} \mathrm{C}$ and assayed for activity.

Biochemical computation by using ligplot and spdbv LIGPLOT (v.4.4.2) is a program for automatically plotting protein-ligand interactions, which automatically generates schematic diagrams of protein-ligand interactions from a given PDB file (Wallace et al., 1995). The interactions were taken into consideration as hydrogen bonds and hydrophobic contacts. Hydrogen bonds are indicated by dashed lines between the atoms involved, while hydrophobic contacts are represented by an arc with spokes radiating towards the ligand atoms they make contact with. The contact atoms are shown with spokes radiating back. The operating manual is located at the site: http://www.biochem.ucl.ac.uk/bsm/ligplot/manual/. Swiss-PdbViewer was used to deduce structural alignments and compare their active sites or any other relevant parts. Swiss-models was used to generate models, as it is possible to thread a protein primary sequence onto a 3D template and get an immediate feedback of how well the threaded protein will be accepted by the reference structure before submitting a request to build missing loops and refine sidechain packing. Swiss-PdbViewer can also read electron density maps, and provides various tools to build into the density. In addition, various modeling tools are integrated and command files for popular energy minimization packages can be generated. (Guex and Peitsch, 1997).

\section{Results and Discussion}

The properties of interfaces in oligomeric enzymes and their influence on catalytic activity can be studied and explained by two independent methods, involving the use of the structural data and kinetics data. Poltorak et al. (1999a, 1999b) reported that, in alkaline phosphatase from different sources, the same results that in both methods are in reasonable agreement. They proposed (Poltorak et al., 1998) that the catalytic reaction rate is a measure of active form concentration under given conditions, e.g. saturation with substrate. The simple thermal dissociation mechanism is:

$$
\mathrm{E}_{2} \leftrightarrow 2 \mathrm{E}_{1} \rightarrow 2 \mathrm{E}_{\text {den }}
$$

(scheme 1)

$\mathrm{E}_{1}$ is a monomer capable of reversible transformation of the initial structure, $\mathrm{E}_{\mathrm{den}}$ is the product of irreversible transformation (under given conditions) of $\mathrm{E}_{1}$. The protein $\mathrm{E}_{\mathrm{den}}$ is unable to reassociate and form a functionally active protein complex $\mathrm{E}_{2}$ (dimer form). Usually, there are several intermediate active ' $\mathrm{m}$ ' forms of dimeric protein.

$$
\begin{aligned}
& \stackrel{\mathrm{E}_{2(\text { native) }} \leftrightarrow}{K_{\text {den }}} \longrightarrow \mathrm{E}_{2}^{(1)} \leftrightarrow \mathrm{E}_{2}^{(2)} \leftrightarrow \mathrm{E}_{2}^{(3)} \ldots \leftrightarrow \mathrm{E}_{2}^{(\mathrm{m})} \stackrel{K_{\text {dis }} \underset{\text { den }}{\longrightarrow}}{\longrightarrow} 2 \mathrm{E}_{1} \\
& \stackrel{\text { (schem }}{\longrightarrow}
\end{aligned}
$$

Poltorak et al. (1999b) proposed that in the event of variations of the rate constants for the reciprocal transitions of various forms of catalytically active protein, $m$, the real number of forms is always greater than a certain quantity $n$ that describes the irreversible transitions of $n$ enzyme dimeric forms with similar rate constants. To determine this value from the kinetic curves it is convenient to introduce the dimensional ratio of period of induction $\tau_{1}$ and period of thermal dissociation $\tau_{2}$ shown in Fig. 1 .

$$
\delta=\frac{\tau_{1}}{\tau_{2}-\tau_{1}}=\mathrm{R}-1
$$

where $\mathrm{R}$ is shown in the ordinate of Fig. 1.

For scheme 2, the exact analytical solution gives that value $\delta$ is depending only of the number of steps before loss of activity $n$. 

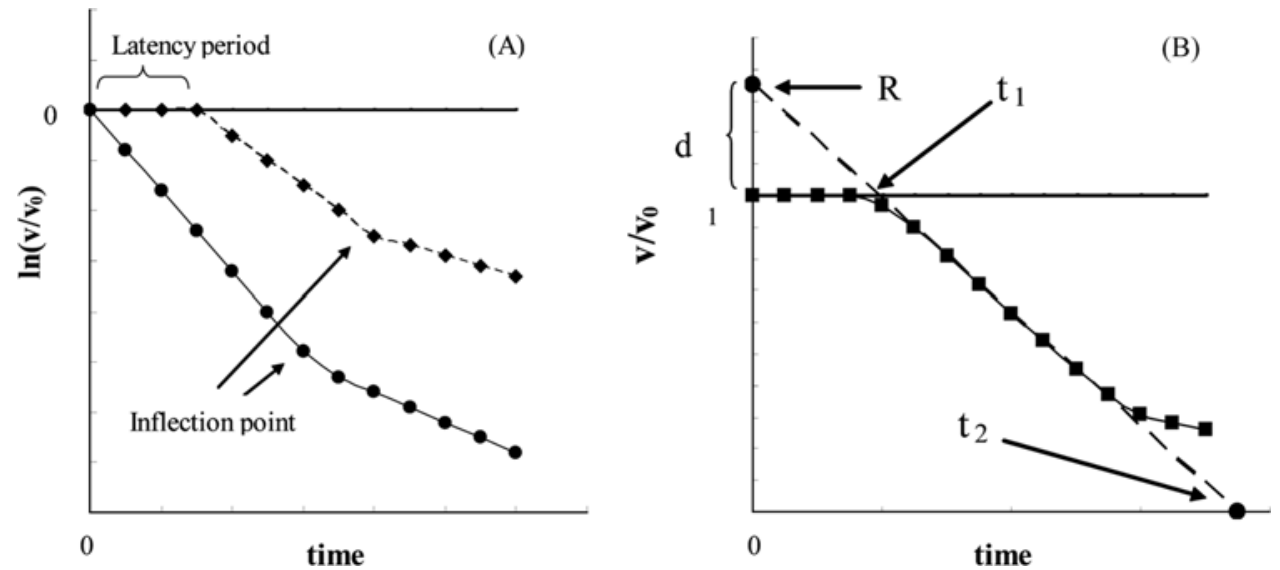

Fig. 1. Typical shapes of thermal dissociation curves for a dimeric enzyme that undergoes thermal dissociation without and with a latency period corresponding to the Scheme 1 and 2 respectively; A) $\boldsymbol{O}$, without latency period; $\boldsymbol{\nabla}$, with latency period; B) The graph shows the method for determining of $\delta, \mathrm{R}$ and $\tau$. (Moosavi-Nejad et al., 2003).

$$
\delta=\mathrm{e}^{-(\mathrm{n}-1)}\left[1+\frac{(\mathrm{n}-1)^{\mathrm{n}-2}}{(\mathrm{n}-2) !}+\Sigma_{\mathrm{m}=1}^{\mathrm{n}=2}\left(\frac{(\mathrm{n}-1)^{\mathrm{m}}}{\mathrm{m} !}\right)-1\right]
$$

where $\mathrm{m}$ is the total transition steps before dissociation of enzyme and $\mathrm{n}$ is the minimum transition steps which may be happened.

However, the exact calculation of $n$ is practically impossible from this expression. For this reason, it was approximated by the empirical equation (Poltorak et al., 1999b)

$$
\delta=\frac{0.13(\mathrm{n}-1)}{1-0.05 \mathrm{n}}
$$

or

$$
\mathrm{n}=\frac{0.13+\delta}{0.13-0.05 \delta}
$$

Such calculation gives only limiting minimal value of $n$ for scheme 2 (Poltorak et al., 1999b). These make it possible to estimate the minimal number of steps for a dimeric protein conversion by dissociation into catalytically inactive monomers. To explain experimental data, Poltorak et al. (1998; 1999a; 1999 b) proposed the phenomenon of "conformational lock" as an interprotein contact with possible partial breaks (loosening of binding between globules). Each successive state of the interprotein binding in an oligomeric enzyme cannot be achieved without the break of the preceding one. It has been shown that in some oligomeric enzymes, such as alkaline phosphatase, several contact areas are separated, and conformational lock can open stepwise through these areas.

The plot of residual activity of SOD versus temperature shows that the activity remained constant at incubation temperatures below $70^{\circ} \mathrm{C}$. However, above $73^{\circ} \mathrm{C}$, the activity rapidly reduces. The $\mathrm{T}_{\text {opt }}$ of SOD is calculated as $73^{\circ} \mathrm{C}$ (see Fig. 2 ).

The $\delta$ and $\mathrm{R}$ values and calculated minimum thermal dissociative steps in the thermal dissociation of SOD based on

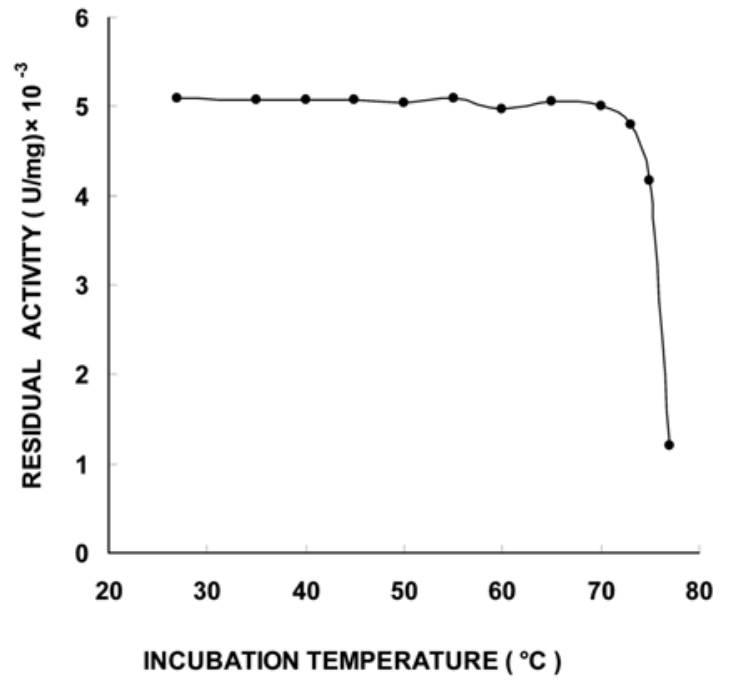

Fig. 2. Residual activity of SOD versus incubation temperature at $0.05 \mathrm{M}$ pH 7.4 Tris- $\mathrm{HCl}$ buffer containing $10^{-4} \mathrm{M}$ EDTA. SOD $\left(4.9 \times 10^{-7} \mathrm{M}\right)$ was incubated at the indicated temperature for 150 minutes followed by rapid cooling to $27^{\circ} \mathrm{C}$ and assayed for residual activity.

scheme 3 was calculated by using the method of Poltorak et al. (1998; 1999a; 1999b) which is shown in Fig. 3. The quantitative values of these parameters show that minimum steps during the dimeric SOD thermal dissocation is two, which is consistent with the results obtained by crystal structure using methods of X-ray (PDB no: 1CBJ). The hypothetical kinetics and processes of thermal dissociation and denaturation of SOD can be described as Scheme 3 .

From the PDB information, SOD from bovine erythrocyte (PDB no:1CBJ) consists of two subunits. Each subunit consists of 151 amino acid residues. There are two contact areas between the two subunits. There is a Greek key eightstranded $\beta$-barrel in each subunit of SOD. This structure is very important for the biological function of SOD (Bourne et 


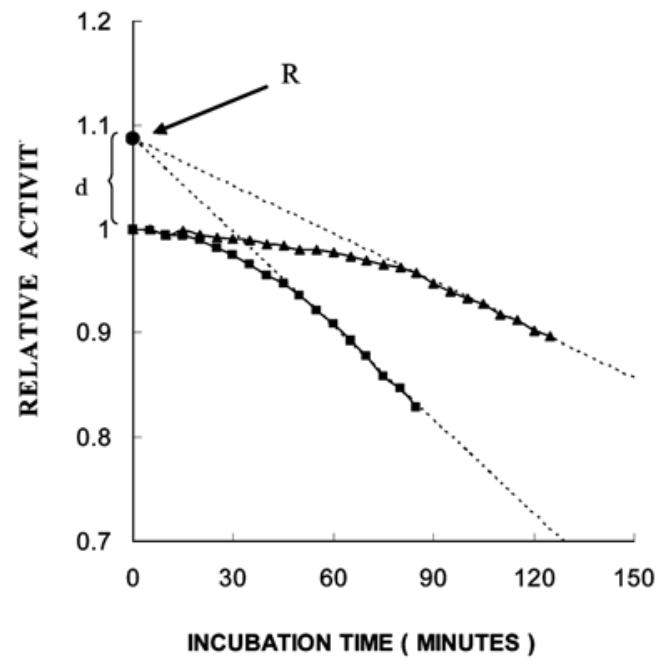

Fig. 3. Kinetics of thermal dissociation of SOD at different initial temperatures: $75^{\circ} \mathrm{C}(\boldsymbol{\Delta}) ; 77^{\circ} \mathrm{C}(\boldsymbol{\square})$. Conditions of thermal dissociation: $[\mathrm{SOD}]_{0}=4.9 \times 10^{-7} \mathrm{M}$ in $0.05 \mathrm{M}$ pH 7.4 Tris- $\mathrm{HCl}$ buffer containing $10^{-4} \mathrm{M}$ EDTA. $\mathrm{R}=1+\delta$ is the ordinate intercept produced by a tangent to the inflection point of the kinetic curve; $\delta$ is a dimensionless parameter used to calculate a minimal number of active intermediate enzyme forms. And $\delta_{75^{\circ} \mathrm{C}}$ $=0.08, \mathrm{n}_{75^{\circ} \mathrm{C}}=1.67 \approx 2 ; \delta_{75^{\circ} \mathrm{C}}=0.09, \mathrm{n}_{77^{\circ} \mathrm{C}}=1.75 \approx 2$.

al., 1996; Battistoni et al., 1998). The Greek key eightstranded $\beta$-barrel consists of $\beta_{1}$ (T2-L8), $\beta_{2}(\mathrm{Q} 15-\mathrm{K} 23), \beta_{3}$ (T26G35), $\beta_{4}(\mathrm{G} 39-\mathrm{Q} 47), \beta_{5}(\mathrm{G} 80-\mathrm{A} 87), \beta_{6}$ (G91-D99), $\beta_{7}(\mathrm{R} 113-\mathrm{E} 119)$, $\beta_{8}(\mathrm{~S} 140-\mathrm{A} 150)$. The contact areas of SOD subunits are shown in the Table 1 and Fig. 4. The amino acid residues at contact areas and their interactions were presented at Figures 4 and 5.

The two subunits of SOD molecule make contact with each other through two identical contact areas by H-bonds and hydrophobic interactions: First, the residue of Ile149 on the subunit A interacts with the residues Gly112 and Gly49 of chain B through H-bonds and Lys151 on subunit A connect with Asp 50 on subunit B through a $\mathrm{H}_{2} \mathrm{O}$ molecule by $\mathrm{H}-$ bonds (see Figs 4 and 5). Second, the residues Val5, Val7, Thr17, Val146, Gly148, Ile149, Ala150 and Lys151 of subunit A interact with residues Phe48, Gly49, Asp50, Asn51, Thr52, Ile111, Gly112 and Arg113 of chain B through hydrophobic interactions (see Figs 4 and 5). When the thermal dissociation occurs, one of two contact areas may be cleaved (first intermediate) at step one, then the second contact area probably cleaved (second intermediate). Finally, the two subunits of SOD can be separated during thermal dissociation or conformational lock, and the dimeric SOD may be dissociated into monomeric structure (see scheme 3). Meanwhile, the spatial structure along the contact area may change. These spatial structural changes, for example, $\beta_{1}, \beta_{4}, \beta_{7}$ and $\beta_{8}$, might induce the reduction or lose of activity of SOD.

His44, His46 and His 118 are located at active site of SOD from bovine erythrocyte, and are very important for the catalytic function of SOD (Hough et al., 2000; Miller, 2004). From the PDB information of SOD (PDB no:1CBJ), His44 and His46 are located on the $\beta_{4}$ (G39-Q47), His118 is located on the $\beta_{7}$ (R113-E119), the spatial structure changes of $\beta_{4}, \beta_{7}$ might cause spatial change of the active site and lead to the reduction or lose of activity of SOD. On the other hand, conformational change of $\beta_{8}$ (S140-A150) may affect the mobility of loops II (residues Glu119-Leu142), and the mobility in the Glu119-Leu142 loop region is very important for catalytic function of $\mathrm{Cu}, \mathrm{Zn}-\mathrm{SOD}$ (Hough and Hasnain, 1999; Hough et al., 2000; Hough and Hasnain, 2003). Moreover, there is a disulfide bridge between Cys144 and Cys55 in each subunit of SOD, and Cys144 is located on $\beta_{8}$

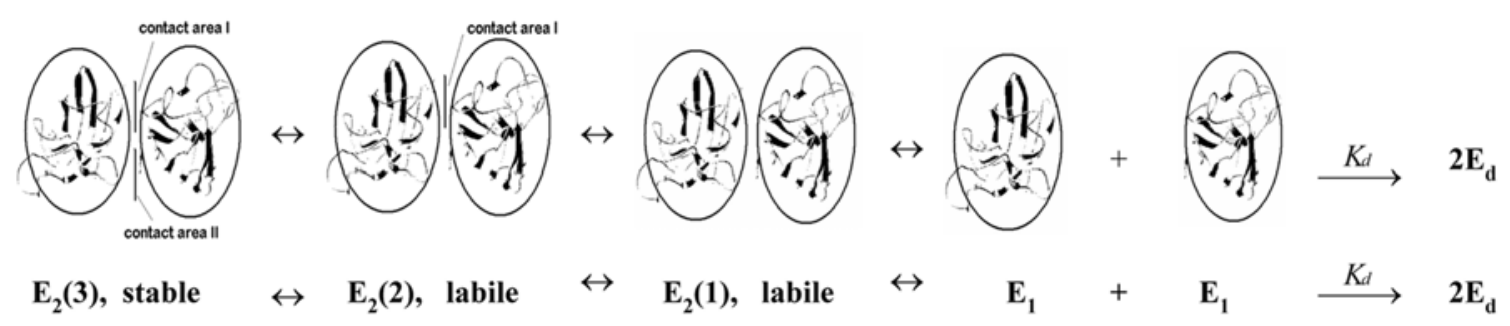

Scheme 3. Hypothetical kinetics and processes of thermal dissociation and denatureation of SOD. Where $\mathrm{E}_{2}$ (3) is the native dimer with two intact contacts, $E_{2}(2)$ and $E_{2}(1)$ are the labile dimer (two intermediates), and $E_{1}$ is the inactive or weak active monomer. $E_{d}$ is the denatured monomer.

Table. 1 Contact areas and residues involved in conformational lock

\begin{tabular}{ccc}
\hline Contact area* & Residues in chain A & Residues in chain B \\
\hline \multirow{2}{*}{ I } & Val5,Val7, Thr17 & Phe48-Gly49-Asp50-Asn51-Thr52, \\
& Val146, Gly148-Ile149-Ala150-Lys151 & Ile111-Gly112-Arg113 \\
II & Phe48-Gly49-Asp50-Asn51-Thr52, & Val5,Val7, Thr17 \\
& Ile111-Gly112-Arg113 & Val146, Gly148-Ile149-Ala150-Lys151 \\
\hline
\end{tabular}

*Conformational lock in SOD consists of two identical contact areas. 

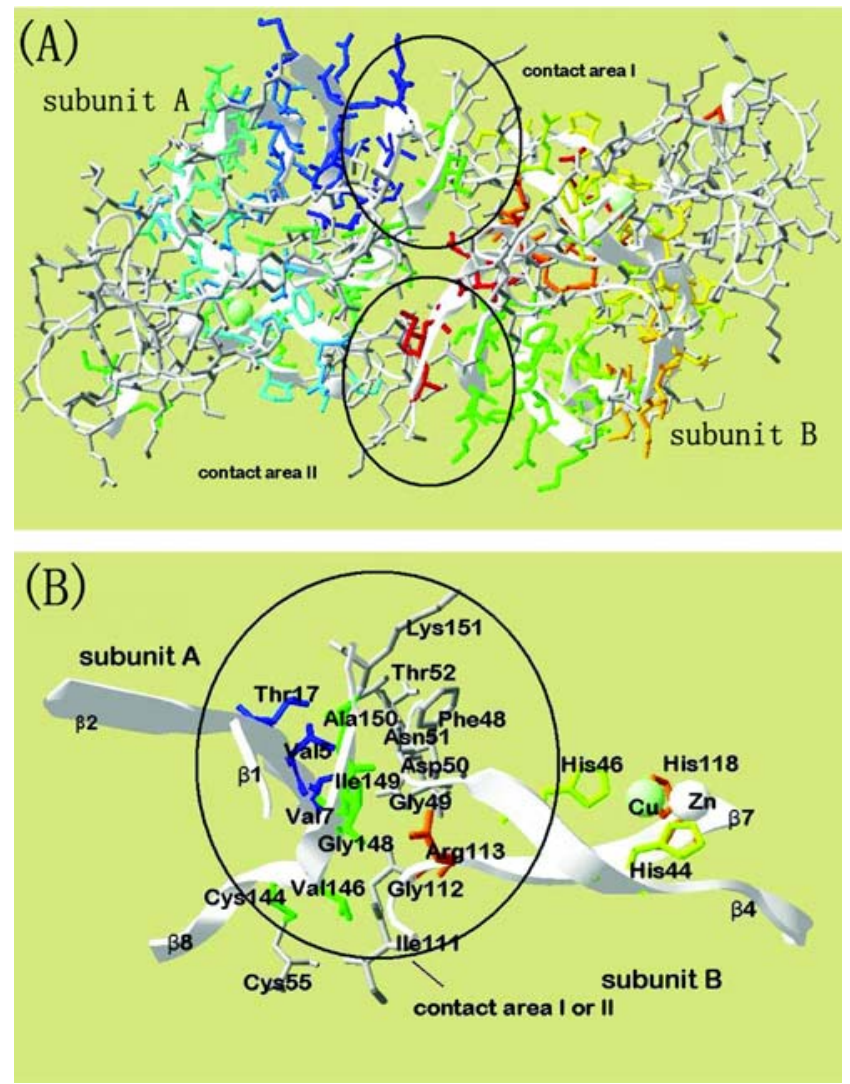

Fig. 4. (A) shows the contact sites of two identical subunits of SOD. (B) shows the expanded of contact area I or II and part of active center. Conformational lock of SOD consists of two identical contact areas. This figure is generated by using the software of swiss-pdbviewerV3.7 (Guex and Peitsch, 1997) downloaded from site: http://www.expasy.org/spdbv.

(S141-A150). It is reported that the intra-subunit disulfide bond is very important for maintaining the spatial structure and compactness of SOD (Abernethy et al., 1974). The spatial structure change of $\beta_{8}$ (S114-A150) may also affect the disulfide bond, which lead to the reduction of compactness and stability of SOD (Abernethy et al., 1974). It can also be seen that residues Val5 and Val7 are located on $\beta_{1}$, while residues Lys151, Ala150, Ile149, Gly148, Val146 are located at $\beta_{8}$. These residues are very close to the C-terminal and $\mathrm{N}$ terminal (see Fig. 4). The disruption of conformational lock and dissociation of contact areas might induce the unfolding and lose of catalytic activity for SOD.

Structural analysis of the dimeric SOD showed that the two identical contact areas between the two subunits of SOD interact with elements of secondary structure of active centers and Greek-key eight-stranded $\beta$-barrel. This may be helpful to maintain the catalytic activity, spatial structure, compactness and stability of SOD, that is in agreement with the works of Rigo et al. (1978) and Bertini et al. (1994) that reported the monomeric SOD displays very low catalytic activity. Dissociation of these contacts might destroy the spatial structure of active

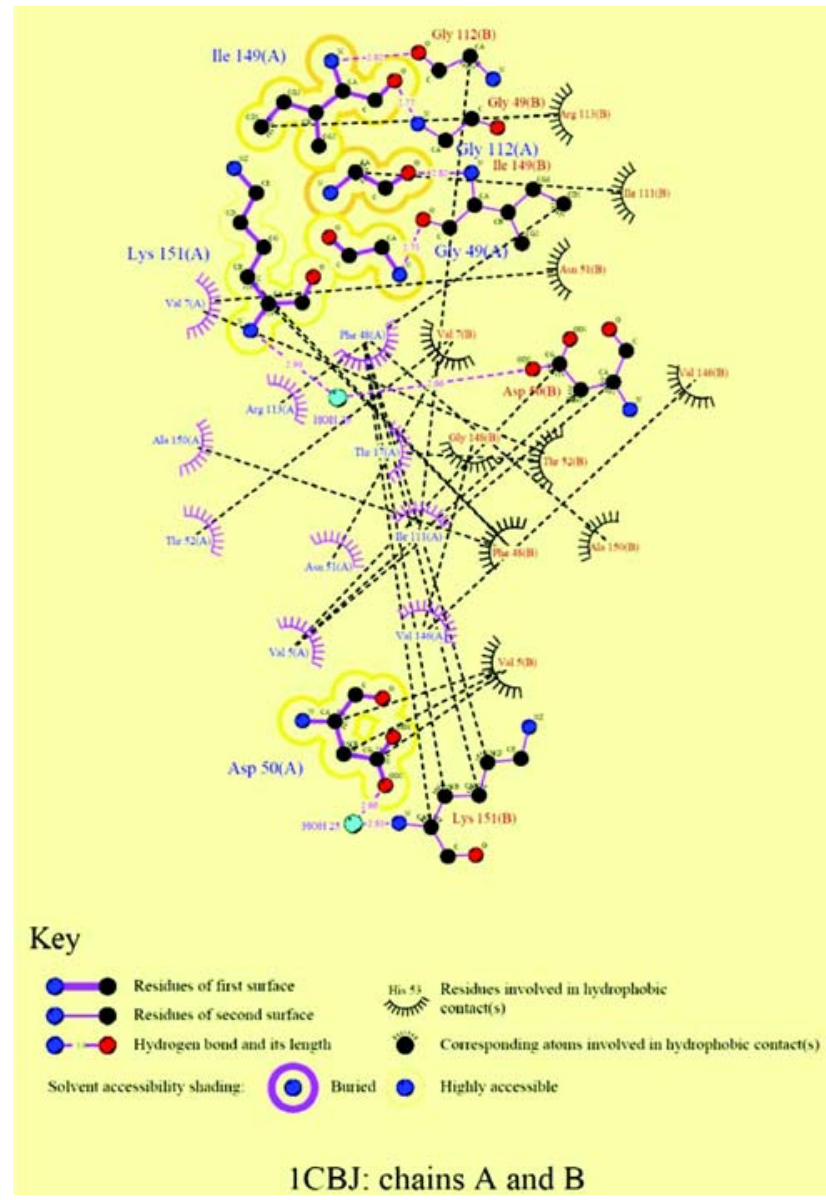

Fig. 5. Interactions of residues of SOD (PDB no: $1 \mathrm{CBJ}$ ) at the contact areas. This figure is generated by using the software of Ligplot (Wallace et al., 1995). The purple dashed lines represent hydrogen bonds, the black dashed lines represent hydrophobic interactions and spiked residues show hydrophobic contacts. Residues in subunit A are shown by purple color and residues in subunit B are shown by black color.

center and the Greek-key eight-stranded $\beta$-barrel. The total number of contacts in the conformational lock is two and this is in good agreement with kinetic data, giving the same minimal number of intermediates during the thermal dissociation of SOD.

Acknowledgments The grant of Third World Academy of Science (TWAS) for Dr. F. C. Chilaka and financial support provided from the Research Council of the University of Tehran and Iran National Science Foundation (INSF) are gratefully acknowledged.

\section{References}

Abernethy, J. L., Steinman, H. M. and Hill, R. L. (1974) Bovine erythrocyte superoxide dismutase. Subunit structure and sequence location of the intrasubunit disulfide bond. J. Biol. 
Chem. 249, 7339-7347.

Bannister, J. V., Bannister, W. H. and Rotilio, G. (1987) Aspects of the structure, function, and applications of superoxide dismutase. CRC Crit. Rev. Biochem. 22, 111-180.

Battistoni, A., Folcarelli, S., Gabbianelli, R., Capo, C. and Rotilio, G. (1996) The $\mathrm{Cu}, \mathrm{Zn}$ superoxide dismutase from Escherichia coli retains monomeric structure at high protein concentration. Evidence for altered subunit interaction in all the bacteriocupreins. Biochem. J. 320, 713-716.

Battistoni, A., Folcarelli, S., Gervoni, L., Polizio, F., Desideri, A., Giartosio, A. and Rotilio, G. (1998) Role of the dimeric structure in $\mathrm{Cu}, \mathrm{Zn}$ superoxide dismutase. pH-dependent, reversible denaturation of the monomeric enzyme from Escherichia coli. J. Biol. Chem. 273, 5655-5661.

Bertini, I., Piccioli, M., Viezzoli, M. S., Chiu, C. Y. and Mullenbach, G. T. (1994) A spectroscopic characterization of a monomeric analog of copper, zinc superoxide dismutase. Eur. Biophys. J. 23, 167-176.

Bordo, D., Djinovic-Carugo, K. and Bolognesi, M. (1994) Conserved patterns in the $\mathrm{Cu}, \mathrm{Zn}$ superoxide dismutase family. J. Mol. Biol. 238, 366-386.

Bourne, Y., Redford, S. M., Steinman, H. M., Lepock, J. R., Tainer, J. A. and Getzoff, E. D. (1996) Novel dimeric interface and electrostatic recognition in bacterial $\mathrm{Cu}, \mathrm{Zn}$ superoxide dismutase. Proc. Natl. Acad. Sci USA 93, 12774-12779.

Desideri, A., Falconi, M., Polticelli, F., Bolognesi, M., Djinovic, K. and Rotilio, G. (1992) Evolutionary conservativeness of electric field in the $\mathrm{Cu}, \mathrm{Zn}$ superoxide dismutase active site. Evidence for co-ordinated mutation of charged amino acid residues. J. Mol. Biol. 223, 337-342.

Forman, H. S. and Fridovich, I. (1973) On the stability of bovine superoxide dismutase. The effects of metals. J. Biol. Chem. 248, 2645-2649.

Getzoff, E. D., Tainer, J. A., Stempien, M. M., Bell, G. I. and Hallewell, R. A. (1989) Evolution of CuZn superoxide dismutase and the Greek key beta-barrel structural motif. Proteins 5, 322-336.

Guex, N. and Peitsch, M. C. (1997) SWISS-MODEL and the Swiss-PdbViewer: An environment for comparative protein modeling. Electrophoresis 18, 2714-2723.

Hough, M. A. and Hasnain, S. S. (1999) Crystallographic structures of bovine copper-zinc superoxide dismutase reveal asymmetry in two subunits: functionally important three and five coordinate copper sites captured in the same crystal. $J$. Mol. Biol. 287, 579-592.

Hough, M. A., Strange, R. W. and Hasnain, S. S. (2000) Conformational variability of the $\mathrm{Cu}$ site in one subunit of bovine $\mathrm{CuZn}$ superoxide dismutase: the importance of mobility in the Glu119-Leu142 loop region for catalytic function. $J$. Mol. Biol. 203, 231-241.

Hough, M. A. and Hasnain, S. S. (2003) Structure of fully reduced bovine copper zinc superoxide dismutase at $1.15 \mathrm{~A}$. Structure 11, 937-946.

Imlay, K. R. C. and Imlay, J. A. (1996) Cloning and analysis of
sodC, encoding the copper-zinc superoxide dismutase of Escherichia coli. J. Bacteriol. 178, 2564-2571.

Kroll, J. S., Langford, P. R., Wilks, K. and Keil, A. D. (1995) Bacterial $[\mathrm{Cu}, \mathrm{Zn}]$-superoxide dismutase: phylogenetically distinct from the eukaryotic enzyme, and not so rare after all. Microbiology 141, 2271-2279.

Liang, Y., Qu, S. S., Wang, C. X., Zou, G. L., Wu, Y. L. and Li, D. H. (2000) An on-line calorimetric study of the dismutation of superoxide anion catalyzed by SOD in batch reactors. Chem.Eng. Sci. 55, 6071-6078.

Luo, G. D., Li, W., Zheng, L. X., Cheng, Y. H., Funakoshi, S. and Yajima, H. (1987) Studies on superoxide dismutase. I. Purification and properties of superoxide dismutase from Azotobacter vinelandii-230. Chem. Pharm. Bull. 35, 42294234.

Marklund, S. and Marklund, G. (1974) Involvement of the superoxide anion radical in the autoxidation of pyrogallol and a convenient assay for superoxide dismutase. Eur. J. Biochem. 47, 469-474.

Miller, A. F. (2004) Superoxide dismutases: active sites that save, but a protein that kills. Curr. Opin. Chem. Biol. 8, 162-168.

Moosavi-Nejad, S. Z., Moosavi-Movahedi, A. A., Rezaei-Tavirani, M., Giovanni, F. and Rosaria, M. (2002) Conformational lock and dissociative thermal inactivation of lentil seedling amino oxidase. J. Biochem. Mol. Biol. 36, 167-172.

Poltorak, O. M., Chukhray, E. S. and Torshin, I. Y. (1998) Dissociative thermal inactivation, stability, and activity of oligomeric enzymes. Biochemistry (Moscow) 63, 303-311.

Poltorak, O. M., Chukhrai, E. S., Kozlenkov, A. A, Chaplin, M. F. and Trevan, M. D. (1999a) The putative common mechanism for inactivation of alkaline phosphatase isoenzymes. J. Mol. Catalysis. B-Enzym. 7, 157-163.

Poltorak, O. M., Chukhray, E. S., Torshin, I. Y., Atyaksheva, L. F., Trevan, M. D. and Chaplin, M. F. (1999b) Catalytic properties, stability and the structure of the conformational lock in the alkaline phosphatase from Escherichia coli. J. Mol. Catalysis. B-Enzym. 7, 165-172.

Rigo, A., Marmocchi, F., Cocco, D., Viglino, P. and Rotilio, G. (1978) On the quaternary structure of copper-zinc superoxide dismutases. Reversible dissociation into protomers of the isozyme I from wheat germ. Biochemistry 175, 534-537.

Segel, I. H. (1995) Effects of $\mathrm{pH}$ and temperature; in Enzyme kinetics, pp. 926-942, John Wiley \& Sons press, New York, USA.

Wallace, A. C., Laskowski, R. A. and Thornton, J. M. (1995) LIGPLOT: A program to generate schematic diagrams of protein-ligand interactions. Protein Engineering 8, 127-134.

Yuan, Z. B. and Gao, R. M. (1997) Kinetics and mechanism of pyrogallol autoxidation. Chemical J. Chinese Universities 18, 1438-1441.

Zou, G. L., Gui, X. F., Zhong, X. L. and Zhu, Y. F. (1986) Improvements in pyrogallol autoxidation method for the determation of SOD activity. Prog. Biochem. Biophys. 4, 7173. 